\begin{tabular}{llll} 
Abstract 237 & Table 1 & & \\
\hline Method & Probe Placement & Aim & Threshold \\
\hline IVC collapsibility/ & Sub-xiphoid area & Fluid & $>50 \% / 12 \%$ \\
distensibility & & responsiveness & \\
Ejection fraction & Parasternal long axis view & Cardiac contractility & $<50 \%$ \\
Lung ultrasound & Intercostals space to count & Extra-vascular lung & $>3$ B lines/ \\
& B lines & water & view \\
\hline
\end{tabular}

tertiary care hospital in Eastern India. The sample size was 56, calculated on the basis of a similar pediatric RCT. Patient enrolment occurred between May 2019 and July 2020. Children aged between 1 month and 12 years with suspected septic shock were randomized to receive either ultrasound or clinically guided fluid boluses (in a 1:1 ratio) and subsequently followed up for primary and secondary outcomes. Exclusion criteria were Dengue, Anaphylaxis, Ascites, and patients with pre-existing chronic kidney disease, interstitial lung disease, heart disease, and adrenal insufficiency. Ultrasound was used in the treatment group whenever there was clinical suspicion of inadequate perfusion.

Results 68 children were enrolled in the study. 4 patients of Dengue and 4 patients who died within 24 hours were excluded. The number (\%) of patients with fluid overload on day 3 of admission was significantly lower in the ultrasound group $(25 \%$ vs. $62 \%, \mathrm{p}=0.012)$ as was the $\mathrm{CFB} \%$ on day 3 $(6.8 \pm 6.6 \%$ vs. $13.4 \pm 10.7 \%, p=0.019)$. Total fluid bolus was significantly lower \{median of $40(30-50) \mathrm{ml} / \mathrm{kg}$ vs. 50 (4080) $\mathrm{ml} / \mathrm{kg}, \mathrm{p}=0.003\}$. Resuscitation time was significantly lower in the ultrasound group $(13.4 \pm 5.6 \mathrm{~h}$ vs. $20.5 \pm 8 \mathrm{~h}$, $\mathrm{p}=0.002)$ and so was the requirement of Furosemide $(39.3 \%$ vs. $71.4 \%, \mathrm{p}=0.016)$. None of the deaths in the ultrasound group were due to unresolved shock $(p=0.101)$. There was no significant benefit derived from ultrasound in terms of ventilator duration, inotrope/vasopressor requirement, length of PICU/hospital stay, and mortality.

Conclusions Due to a lack of comparative studies on this topic, our study adds insight into the utility of ultrasound in improving outcomes in septic shock. Ultrasound made the PICU physician exercise restraint in administering fluid boluses and allowed earlier initiation of inotropes. Ultrasound was found to be significantly better than clinically guided therapy, in preventing fluid overload as well as being quicker in achieving initial hemodynamic stabilization. Hence ultrasound is a potentially useful tool for fluid resuscitation in children with septic shock.

\section{USE OF TELEMEDICINE IN A DEVELOPMENTAL AND BEHAVIOURAL PAEDIATRIC SERVICE DURING THE COVID-19 PANDEMIC:INITIAL EXPERIENCES OF CAREGIVERS \& PROVIDERS, SINGAPORE}

Mae Yue Tan, Ying Qi Kang, Shang Chee Chong, Mulay Kalyani Vijayakumar, Tammy Su Hui Lim, Aishworiya Ramkumar. Singapore

\subsection{6/bmjpo-2021-RCPCH.5}

Background Telemedicine has been used increasingly as an alternative care model in the Coronavirus 2019 pandemic. The practice of Developmental and Behavioural Paediatrics (DBP) relies heavily on direct child observations and building trust and rapport with caregivers over time and telemedicine was not routinely used in the Singapore setting.

Objectives We aimed to describe the initial experiences of caregivers and providers with telemedicine in a DBP service in Singapore during this pandemic.

Methods This cross-sectional survey included caregivers who accessed telemedicine (through telephone or video-based platforms) and healthcare providers at a tertiary DBP clinic between 8 April and 14 May 2020, coinciding with the Circuit Breaker (similar to a lockdown) period in Singapore. Participants completed a questionnaire on experiences, perceived benefits and limitations of teleconsultation. Descriptive statistics and tests of comparison were conducted.

Results Around half of the patients ( $\mathrm{N}=216,47.3 \%)$ who were offered a teleconsultation agreed to it, of which 105 caregivers $(48.6 \%)$ participated. Caregivers had children with diagnosis including autism spectrum disorder (33.3\%), language delay (28.6\%), and attention deficit hyperactivity disorder (12.4\%). Most caregivers felt that their concerns were adequately addressed (98.1\%), with good technological connectivity (95.2\%). Majority had no difficulty preparing for the teleconsultation (61.0\%) and $44.8 \%$ reported ease of followup with plans after. Perceived benefits included safety (47.6\%) and reduced need to take time off work (24.8\%). Perceived benefits included safety $(47.6 \%)$ and reduced time off work (24.8\%). Caregivers who had video-based teleconsultation (compared to telephone-based) had more difficulties preparing for the teleconsultation $(52.3 \%$ vs $27.4, p=0.03)$. These caregivers were also more likely to have difficulties with remembering and following up with plans discussed during the consultation, compared to those who had a telephone-based consultation $(52.3 \%$ vs $25.0 \%$, p value $=0.02)$.

Twenty-five providers participated. Providers included paediatricians, allied health therapists e.g. psychologists, speech and occupational therapists and social workers. Fifty-two percent of providers were as satisfied with teleconsultation compared to in person consults. Only $28.0 \%$ of providers rated technology infrastructure to be good. Most providers (92.0\%) felt safer with teleconsultation. The most common difficulty perceived by providers was incompleteness of consultation without assessing the child physically. The two main perceived benefits of teleconsultation by providers included feeling safer as they were able to minimize physical interaction with patients and being able to provide similarly good care with less inconvenience to patients.

Overall $63.8 \%$ of caregivers and $56.0 \%$ of providers would continue teleconsultation services post-pandemic. Amongst providers, paediatricians were more likely to continue teleconsultation services after current restrictions were lifted, compared to non-physician providers $(100 \%$ vs $38.9 \%, \mathrm{p}=0.008)$.

Conclusions Telemedicine was generally well received by caregiver and physician providers. Teleconsultation in a DBP practice setting is a viable option in a pandemic situation and can be considered even beyond this pandemic for continued care.

\section{TRENDS OF NEONATAL SEPSIS AMONG PRETERM INFANTS BORN <32 WEEKS GESTATION OVER A 13- YEAR PERIOD}

Nadira Shahrul Baharin, Guan Lin Goh, Rowena BDela Puerta, Kathleen Tan Zi Xuan, Victor Samuel Rajadurai, Kee Thai Yeo. Singapore

10.1136/bmjpo-2021-RCPCH.6 
Background In spite of recent improvements in the outcomes of preterm infants, sepsis remains to be an important cause of mortality and morbidity. Studies have noted the rise of Gramnegative infections as causes of sepsis in the preterm population.

Objectives We aim to describe the epidemiology, trends and microbial distribution of neonatal sepsis episodes among preterm infants born $<32$ weeks gestation over a 13-year period.

Methods This is a retrospective single-centre cohort study of culture-confirmed neonatal sepsis episodes, which is defined as the isolation of pathogenic organism from blood and/or cerebrospinal fluid cultures. We included newborns that were admitted to the Neonatal Intensive Care Unit (NICU) who were $<32$ weeks birth gestation and birthweight of $\leq 1500 \mathrm{~g}$. Neonatal sepsis episodes were stratified into early-onset sepsis (EOS) occurring at $<72$ hours of birth and late-onset sepsis (LOS) occurring $>72$ hours after birth up till discharge.

Results A total of 2019 preterm infants were included in the study. The characteristics of the cohort: $52.7 \%$ male, median birth gestational age 28 weeks, $19.9 \%$ small-for-gestational age, $89.9 \%$ received antenatal steroids, $85.5 \%$ had a central venous catheter. Over the study period, 263 infants had a total of 273 episodes of culture-confirmed sepsis -49 EOS and 224 LOS episodes. Incidence of sepsis in this cohort decreased from a high of 24.2\% in 2006 to 7.4\% in 2017. This was concordant with a decrease in LOS over the same period: $16.8 \%-21.6 \%$ from $2005-2006$ to $4.9 \%$ in 2017 . EOS incidence remained relatively stable, ranging from 04.4\%. Gram negative bacteria were the predominant pathogen group isolated, accounting for $186 / 281(66.2 \%)$ of all organisms- $40 / 49(81.6 \%)$ of EOS and 146/224 (65.2\%) of LOS episodes. E Coli [24/49 (49.0\%)] and Klebsiella spp [39/224 $(17.4 \%)]$ were the most common microbial causes of EOS and LOS episodes respectively. Coagulase negative staphylococcus (CONS) accounted for 36/224 (16.1\%) of LOS. Fungal infections were predominantly due to Candida spp - 12.1\% (27/ 224 ) of LOS. Of note, there were no fungal infections detected over the recent 5 years.

Conclusions The decrease in the overall incidence of neonatal sepsis among preterm infants $<32$ weeks in our centre over the 13-year study period, is due to a reduction in LOS. Gram-negative bacterial organisms are the predominant infecting organism in this population with E.Coli and Klebsiella spp being the most common causes of EOS and LOS.

\section{KNOWLEDGE, ATTITUDES AND EXPECTATIONS OF PERINATAL CARE DURING THE COVID-19 PANDEMIC}

Samantha Goh, Caleb Lim, Juin Yee Kong, Kee Thai Yeo, Manisha Mathur, Ka-Hee Chua. Singapore

10.1136/bmjpo-2021-RCPCH.7

Background There is limited evidence regarding the transmission SARS-CoV-2 from mother to infant, as well as the optimal management of infected women and infant during pregnancy, labor and early postnatal period. The knowledge, concerns and expectations of women with regards to perinatal and neonatal care during this current COVID-19 pandemic is currently unclear.

Objectives This study aimed to investigate the knowledge and expectations of pregnant women on perinatal and neonatal care during the coronavirus disease 2019 (COVID-19) pandemic in Singapore.

Methods A cross-sectional survey was administered via a secure online platform to pregnant women attending the antenatal clinics between August 2020 and September 2020. Participants aged $>21$ years who were pregnant and had no history of confirmed COVID-19 were included in the study. The survey consisted of 10 questions formulated to evaluate the knowledge and expectations of these women on the perinatal and neonatal care during this current pandemic.

Results A total of 313 pregnant women completed the survey during the study period. The mean age of the participants was 30 years (SD 4; range 22-43 years). The median gestational age of women at survey participation was 25 weeks (range 4-40 weeks). The participants were predominantly multiparous (54\%) and almost all (98\%) had completed secondary level education. Majority of participants were aware of the spread of COVID-19 by respiratory secretions and contact (90\%), and the importance of hand hygiene and face masking (94\%). Up to $72 \%$ agreed or strongly agreed that in-utero transmission of SARS-CoV-2 to the unborn foetus was possible. Most were unsure of the optimal mode of delivery $(77 \%)$ and only $22 \%$ believed that breastfeeding was safe in a pregnant women with active COVID-19. There was no significant association between the sociodemographic factors evaluated and maternal agreement with the possibility of in-utero SARSCoV-2 transmission and the risk associated with vaginal delivery in women with COVID-19. Although 46\% of participants were concerned about the increased risk of contracting COVID-19 during routine clinic appointments at the hospital, only $37 \%$ of the cohort were agreeable with teleconferencing of clinic appointments. More than half $(56 \%)$ of the participants reported that their postnatal confinement plans were affected by the current pandemic.

Conclusions Our survey revealed that majority of participants were aware of modes of transmission and the prevention strategies of SARS-CoV-2, there were however significant gaps identified in their knowledge related to the risk of in-utero transmission and safety of breast feeding along with significant variability to the agreement with alterations to the perinatal care. For best practice we recommend provision of evidence based information early to expectant mothers by the healthcare professionals to reduce misinformation and anxiety amongst pregnant women related to the current pandemic.

\section{HEALTH STATUS OF CHILDREN WITH DOWN SYNDROME IN MYANMAR}

Khine Mi Mi Ko, Khine Mi Mi Ko, Kyaw Linn, Aye Mya Min Aye, Mon Thander Saw, Pyae Phyo Aung. Myanmar

\subsection{6/bmjpo-2021-RCPCH.8}

Background Down Syndrome children have complex healthcare needs. There was no previous data of Pediatric Down Syndrome (PDS) in Myanmar and little is known about their health status and challenges to seek proper screening tests and health care services. Since there are no established local guidelines for PDS, limited resources and inaccessibility to specialty centers especially for those form remote areas, more health care challenges are encountered throughout the country.

Objectives To assess the health status, screening tests and follow up conditions of Myanmar Down Syndrome children 